\title{
SOLAR-v: A new solar spectral irradiance dataset based on SOLAR/SOLSPEC observations during solar cycle 24
}

\author{
M. Meftah ${ }^{1}$, M. Snow ${ }^{2}$, L. Damé ${ }^{1}$, D. Bolseé ${ }^{3}$, N. Pereira ${ }^{3}$, G. Cessateur ${ }^{3}$, S. Bekki ${ }^{1}$, P. Keckhut $^{1}$, A. Sarkissian ${ }^{1}$, \\ and A. Hauchecorne ${ }^{1}$ \\ ${ }^{1}$ LATMOS, Sorbonne Université, Université Paris-Saclay, Université de Versailles Saint-Quentin-en-Yvelines, IPSL, CNRS, \\ 4 place Jussieu, 75252 Paris Cedex 05, France \\ e-mail: Mustapha.Meftah@latmos.ipsl.fr \\ ${ }^{2}$ Laboratory for Atmospheric and Space Physics (LASP), University of Colorado, 1234 Innovation Dr., Boulder, CO 80303, USA \\ 3 Royal Belgian Institute for Space Aeronomy (BIRA-IASB), Brussels, Belgium
}

Received 14 May 2020 / Accepted 20 October 2020

\begin{abstract}
Context. Solar spectral irradiance (SSI) is the wavelength-dependent energy input to the top of the Earth's atmosphere. Solar ultraviolet (UV) irradiance represents the primary forcing mechanism for the photochemistry, heating, and dynamics of the Earth's atmosphere. Hence, both temporal and spectral variations in solar UV irradiance represent crucial inputs to the modeling and understanding of the behavior of the Earth's atmosphere. Therefore, measuring the long-term solar UV irradiance variations over the 11-year solar activity cycle (and over longer timescales) is fundamental. Thus, each new solar spectral irradiance dataset based on long-term observations represents a major interest and can be used for further investigations of the long-term trend of solar activity and the construction of a homogeneous solar spectral irradiance record.

Aims. The main objective of this article is to present a new solar spectral irradiance database (SOLAR-v) with the associated uncertainties. This dataset is based on solar UV irradiance observations $(165-300 \mathrm{~nm})$ of the SOLAR/SOLSPEC space-based instrument, which provides measurements of the full-disk SSI during solar cycle 24.

Methods. SOLAR/SOLSPEC made solar acquisitions between April 5, 2008 and February 10, 2017. During this period, the instrument was affected by the harsh space environment that introduces instrumental trends (degradation) in the SSI measurements. A new method based on an adaptation of the Multiple Same-Irradiance-Level (MuSIL) technique was used to separate solar variability and any uncorrected instrumental trends in the SOLAR/SOLSPEC UV irradiance measurements.

Results. A new method for correcting degradation has been applied to the SOLAR/SOLSPEC UV irradiance records to provide new solar cycle variability results during solar cycle 24. Irradiances are reported at a mean solar distance of 1 astronomical unit (AU). In the 165-242 nm spectral region, the SOLAR/SOLSPEC data agrees with the observations (SORCE/SOLSTICE) and models (SATIRE-S, NRLSSI 2) to within the 1-sigma error envelope. Between 242 and $300 \mathrm{~nm}$, SOLAR/SOLSPEC agrees only with the models.
\end{abstract}

Key words. Sun: general - Sun: fundamental parameters - Sun: UV radiation - solar-terrestrial relations

\section{Introduction}

Solar spectral irradiance (SSI) represents the dominant source of energy to the climate system (Kren et al. 2017). Variations in SSI drive short-term changes in the middle atmosphere and also influence the climate on long timescales (Floyd et al. 2002; Gray et al. 2010; Ermolli et al. 2013; Solanki et al. 2013; Shindell et al. 2020). Ozone creation and destruction is also driven by the ultraviolet (UV) portion of SSI (Heath \& Schlesinger 1986; Haigh 1994). Accurate measurements of the spectral region from 180 to $300 \mathrm{~nm}$ are needed as inputs for atmospheric models that include ozone. The SSI was identified as an essential climate variable by the Global Climate Observing System (GCOS), meaning that long-term measurement is essential for the understanding of past and present climate and the prediction of future climate (Blunden \& Arndt 2014). Longer wavelength visible and nearinfrared solar irradiance penetrate through to the troposphere and the Earth's surface. The visible and near-infrared solar irradiance plays a role in the global climate. All of the UV SSI at wavelengths between 150 and $300 \mathrm{~nm}$ are deposited in the middle atmosphere. This energy plays an essential role in the chemistry, influence, and dynamics of this atmospheric layer. The SSI at wavelengths short of $180 \mathrm{~nm}$ drive the physical state of the upper atmosphere and ionosphere. Accurate measurements of the SSI and their temporal variations are of primary interest to better understand the links between solar variability and climate. Solar irradiance at wavelengths $(\lambda)$ of less than $300 \mathrm{~nm}$ is absorbed by the Earth's atmosphere, so observations at these wavelengths must be performed from the upper atmosphere or from space. During part of solar cycle 24 (December 2008 to late 2019), two independent sets of space-based instruments carried out continuous measurements of the solar spectrum over extended wavelength ranges. The first set of instruments consists of the SOLar STelar Irradiance Comparison Experiment (SOLSTICE; 115-320 nm) and the Spectral Irradiance Monitor (SIM; 300-2400 nm) instruments onboard the SOlar Radiation and Climate Experiment (SORCE) satellite (McClintock et al. 2005; Harder et al. 2005; Rottman 2005). Measurements were carried out between May 2003 and February 2020. A second independent set consisting of the SOLar SPECtrometer (SOLSPEC) instrument (Thuillier et al. 2009) of the SOLAR payload on board the International Space Station (ISS) performed 


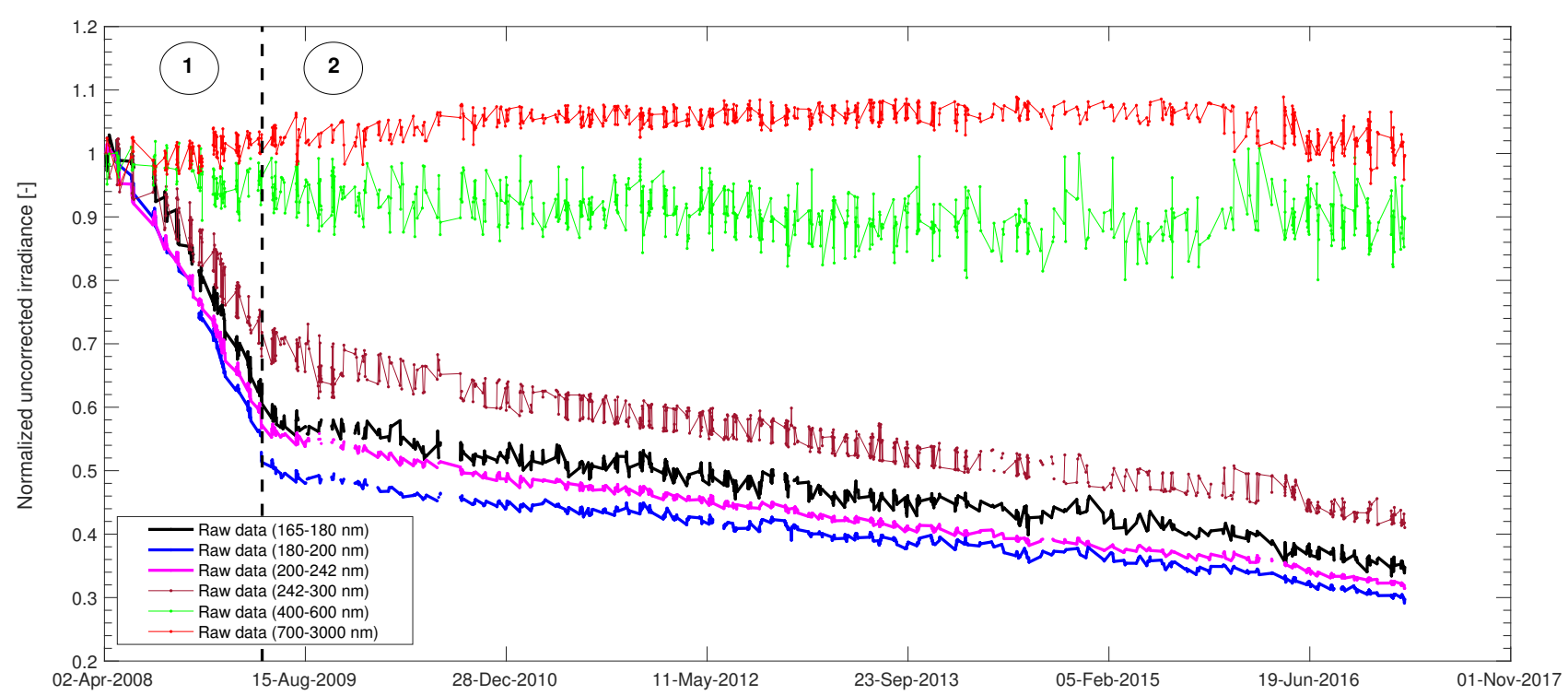

Fig. 1. Evolution of SOLAR/SOLSPEC normalized irradiance as a function of time for different wavelengths (raw data without degradation correction). Symbol (1) locates the first observation period (use of in-flight calibration lamps). Symbol (2) locates the second observation period of the SOLAR/SOLSPEC mission (non-use of in-flight calibration lamps).

accurate measurements of the SSI (165-3000 $\mathrm{nm}$ ) between April 5, 2008 and February 10, 2017 (Meftah et al. 2016, 2018, 2020; Bolsée et al. 2017). The main problem encountered by the solar instruments in space is related to on-orbit degradation processes due to the harsh space environment (BenMoussa et al. 2013; Meftah et al. 2017a) such as atomic oxygen, extreme ultraviolet radiation, charged particles, debris, and temperature extremes. Figure 1 shows the impact of the space environment on the SOLAR/SOLSPEC measurements and illustrates an example of on-orbit degradation of a space-based instrument and understanding of the degradation processes (Meftah et al. 2017a). The SOLAR/SOLSPEC instrument's operating modes that are typically driven by electrical power also have an effect on the degradation process. They introduce a modification of the instrument's temperature field causing a different dynamic of outgassing and contamination of the instrument.

SOLAR/SOLSPEC used many different configurations during its time in orbit, including the following: a nominal solar mode $(\sim 47 \mathrm{~W})$ with adjustment to the measurement exposure time that implied an increase in the duration of an observation sequence (from 10 to $14 \mathrm{~nm}$ in November 2008, from 14 to $18 \mathrm{~nm}$ in October 2015); a calibration mode ( $54 \mathrm{~W})$ using one hollow cathode (HC) lamp providing lines from Argon, Zn, and $\mathrm{Cu}$ to measure the slit function and the dispersion law; a calibration mode $(\sim 58 \mathrm{~W})$ using four tungsten-ribbon lamps $(\mathrm{W})$ for the VIS and IR spectrometers' calibrations; and a calibration mode ( $\sim 84 \mathrm{~W}$ ) using two deuterium lamps (D2) for the UV spectrometer calibration.

Between April 5, 2008 and April 29, 2009 (first observation period: symbol (1) in Fig. 1), D2 and W lamps were used for checking the SOLAR/SOLSPEC instrument stability with time. The light from these calibration sources was carried out using optical fibers, mirrors, and lenses. Degradation in the visible and IR domains is about a few percent (Fig. 1), and can be corrected for by measuring the transmission of the quartz plates and by using the $\mathrm{W}$ lamps. As expected, the degradation in the UV is significant. There is also a transmission loss of the most frequently used quartz plate. The instrument responsivity change is derived by comparing the transmission of the second quartz plate (infrequently used), direct quartz-plate transmission measurements, and D2 lamp data. After April 29, 2009 (second observation period: symbol (2) on Fig. 1), the lamps were no longer used due to a failure of the D2 lamp's power-supply. From that date onwards, the degradation process evolved differently and more slowly. This highlighted an amplification mechanism of the degradation processes and contamination linked to the temperature of the lamps. After April 29, 2009, a new correction must be implemented to correct the SOLAR/SOLSPEC raw data.

We propose a new method based on the Multiple SameIrradiance-Level (MuSIL) technique (Woods et al. 2018). The MuSIL technique uses proxies of solar irradiance such as the magnesium II core-to-wing ratio (Heath \& Schlesinger 1986) to estimate time periods where the measured SSI should be equal. MuSIL analysis determines a correction factor that should be applied to the SSI to bring it into agreement with the variability of the proxy. The SOLAR/SOLSPEC time frame goes from one solar minimum almost to another, so we select time intervals during the rising and declining phases of solar cycle 24. Rather than the "super proxy" used in Woods et al. (2018), we use only the Mg II index from the University of Bremen ${ }^{1}$. This composite is also described in Snow et al. (2014). In this analysis, we concentrate on the UV portion of the SOLAR/SOLSPEC dataset. It has the highest variability, and it can be directly compared to the data from SORCE/SOLSTICE. In this wavelength range, the Mg II index is the best proxy (Dudok de Wit et al. 2009). Other space-based instruments have used the proxy technique to correct instrument degradation such as the Ozone Monitoring Instrument (OMI; Marchenko et al. 2016). A comprehensive comparison of SSI measurements corrected by different techniques to SSI models is given in Coddington et al. (2019). We adapted the Woods et al. (2018) technique in several ways. We carried out our fittings over a solar maximum rather than across a solar minimum, using only a single proxy, and iterating the

\footnotetext{
1 http://www . iup.uni-bremen .de/gome/solar/MgII_ composite.dat
} 
linear fitting (Sect. 2.3). Therefore, we refer to this technique as "modified MuSIL".

This paper outlines the creation of a new SSI dataset based on the SOLAR/SOLSPEC observations that extended from April 2008 to February 2017. We adopt the name SOLAR-v for this dataset since the goal is to extract the relative variability from the SOLAR/SOLSPEC data rather than the absolute irradiance. After correcting the relative changes due to degradation, we use the first-light of SOLAR/SOLSPEC observations to calibrate SOLAR-v back onto an absolute scale. We first describe the analysis technique to uncouple solar-cycle variability and any uncorrected instrumental trends. Finally, we present the new SOLAR-v (Version 1.0) climate data record for solar spectral irradiance between April 2008 and February 2017 with associated wavelength and time-dependent uncertainties. SOLAR-v is then compared with other solar spectral irradiance space-based measurements and models.

\section{The SOLAR/SOLSPEC data-correction method}

SOLAR/SOLSPEC is an absolutely calibrated spectroradiometer measuring the SSI over an extended wavelength range $(165-3000 \mathrm{~nm})$ thanks to three double monochromators: UV, VIS, and IR. The absolute calibration was carried out using the spectral irradiance standards, such as the blackbody of the Physikalisch-Technische Bundesanstalt (PTB; Braunschweig, Germany). SOLAR/SOLSPEC made solar acquisitions between April 5, 2008 and February 10, 2017. Uncorrected irradiance (Fig. 1) is obtained from Eq. (1), where all the corrections are taken into account except the instrumental degradation:

$\operatorname{SSI}_{u}(\lambda, t)=\left(S_{n}(\lambda, t)-\frac{\langle\mathrm{DC}(t)\rangle}{\tau(t)}\right) \times \frac{R(\lambda, t)}{k(\lambda, t, T)} \times\left(\frac{z(t)}{1 \mathrm{AU}}\right)^{2}$,

where $\operatorname{SSI}_{u}(\lambda, t)$ is the uncorrected irradiance (instrument degradation is not taken into account) as measured by SOLAR/SOLSPEC at a given wavelength $(\lambda)$ and for a given time $(t) . S_{n}(\lambda, t)$ (in counts $\mathrm{s}^{-1}$ ) is the linearized signal provided by the detector. $\langle\mathrm{DC}(t)\rangle$ (in counts) is the mean dark current of the detector taken before and after a solar spectrum measurement during the integration time ( $\tau(t)$ in $\mathrm{s}) . R(\lambda, t)$ (in $\mathrm{mW} \mathrm{m}^{-2} \mathrm{~nm}^{-1}$ counts $^{-1} \mathrm{~s}$ ) represents the absolute responsiveness of the instrument. $k(\lambda, t, T)$ is a temperature correction. $z(t)$ (in $\mathrm{km}$ ) is the distance between SOLAR/SOLSPEC and the Sun. The astronomical unit (1 AU) is equal to $149597870.700 \mathrm{~km}$.

Equation (1) depends on engineering corrections and improved calibrations, which are explained in detail by Meftah et al. $(2016,2017 \mathrm{~b}, 2018)$. Methodology to obtain contributions in the uncertainty budget of the SOLAR/SOLSPEC SSI measurements is explained in Bolsée et al. (2017) and Meftah et al. (2018).

The method to correct SOLAR/SOLSPEC data from degradation is described below.

\subsection{Preparation of the SOLAR/SOLSPEC data}

The raw SOLAR/SOLSPEC data have several artifacts that need to be removed before the modified MuSIL technique can be applied. In particular, the rapid degradation during the early observations shown in Fig. 1 has a very different slope to the data of the second period after the SOLAR/SOLSPEC calibration lamps were no longer used.

Since the degradation rate is much larger than the variation due to solar variability, a pair of simple linear fits characterizes the uncorrected data in epochs (1) and (2) well. Before fitting a linear trend to the data, we removed daily observations that can be considered statistical outliers. Figure 1 shows the data after outliers have been removed. We iteratively fit a linear function and removed points whose residuals are more than two standard deviations away from zero, then we fit a new linear function to the observations. We took the data in epoch (2) as the baseline, and adjusted epoch (1) to match the linear trend of epoch (2).

Adjusting the trend of the uncorrected early epoch data with the modified MuSIL technique will extract relative variations in the data occurring since the launch. Our correction of the trend in epoch (1) does not preserve the absolute calibration, but using the first-light SOLSPEC data to correct the absolute scale preserves the preflight calibration.

After the outlier removal and adjusting the slope of the early epoch, we interpolated the SOLAR/SOLSPEC data to a daily cadence. Figure 2 a shows the data after preparation.

\subsection{Preparation of the proxy}

Similarly to the Woods et al. (2018) analysis, we scaled the proxy to a range from 0-100 (Fig. 2b). In our analysis, we used only one proxy, the Bremen Mg II composite (Snow et al. 2014). After scaling, we then smoothed the proxy time series with a 180-day boxcar to remove solar rotational variability and preserve only the solar-cycle variation. This smoothing is important because SOLAR/SOLSPEC did not take a daily measurement (Thuillier et al. 2009; Meftah et al. 2016), and therefore it did not adequately sample many solar rotations. Keeping solar rotational variability in the proxy would add noise to the fits later in the analysis (Sect. 2.3). We then truncated the proxy time series to include only the times overlapping with the SOLAR mission.

The next step in the modified MuSIL technique is to identify the levels in the proxy used (Fig. 2b). Our strategy is to select levels that occur in the proxy as far apart in time as possible. This provides the greatest leverage in correcting trends in the SOLAR/SOLSPEC data. We also chose levels that span the variability of solar cycle 24 to improve the accuracy of the technique. In Woods et al. (2018), the slopes determined from different proxy levels are not all the same for any given instrument. If they were, then selecting a single proxy level would be sufficient. In the case of real measurements (Fig. 1), what appears to be a linear trend is slightly nonlinear.

The final strategy in selecting proxy levels is to choose levels on the steep slopes of the rising and declining phases. A small range of scaled proxy values $( \pm 1)$ then defines a small range of appropriate times. In Sect. 2.3, the uncorrected irradiance is fit with a linear function. The best fit to a line is two points. The second best is two small clusters of points separated in time. That produces the lowest uncertainty in the slope of the fit.

The MuSIL and modified MuSIL techniques use piecewise linear functions to approximate a nonlinear degradation trend. The proxy levels chosen for this project are shown in Fig. $2 b$.

Once the levels of the proxy have been chosen, it's a straight process to determine the dates when those "same irradiance levels" occur. There will be two clusters of irradiance times for each proxy level. Figure $2 \mathrm{~b}$ shows the scaled proxy levels that we chose (20 (pink), 30 (blue), and 40 (green)). Figure 2c shows the SSI data corresponding to the proxy levels as circles.

\subsection{Fitting SSI data to proxy levels}

The fundamental assumption in the modified MuSIL method is that the degradation function is close to linear. We took the SSI 


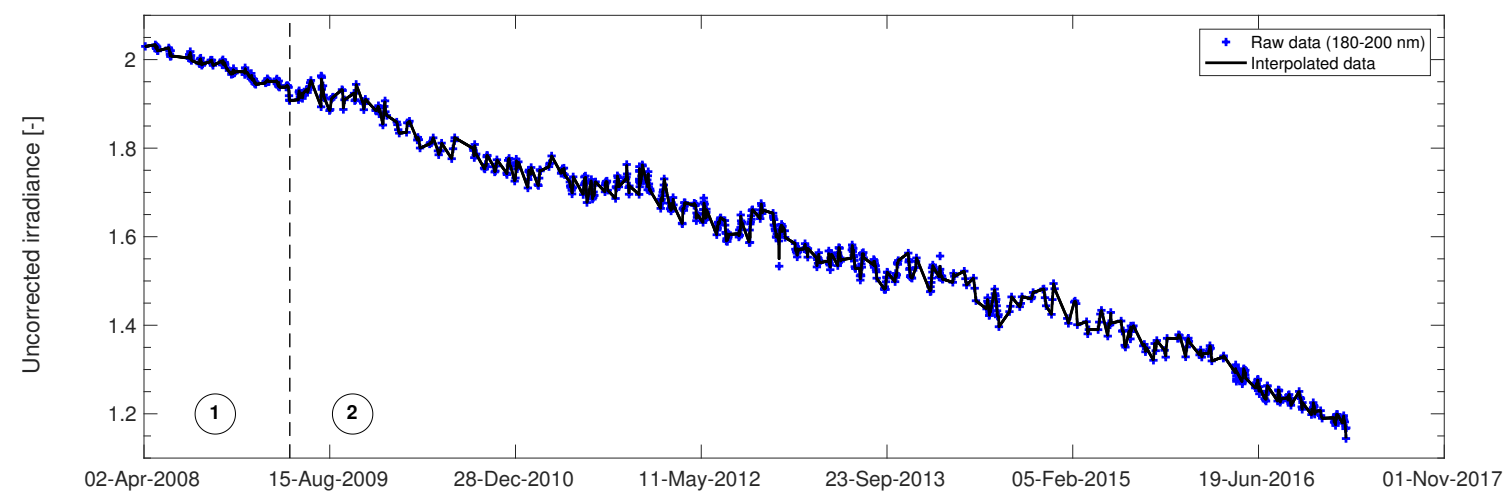

(a)

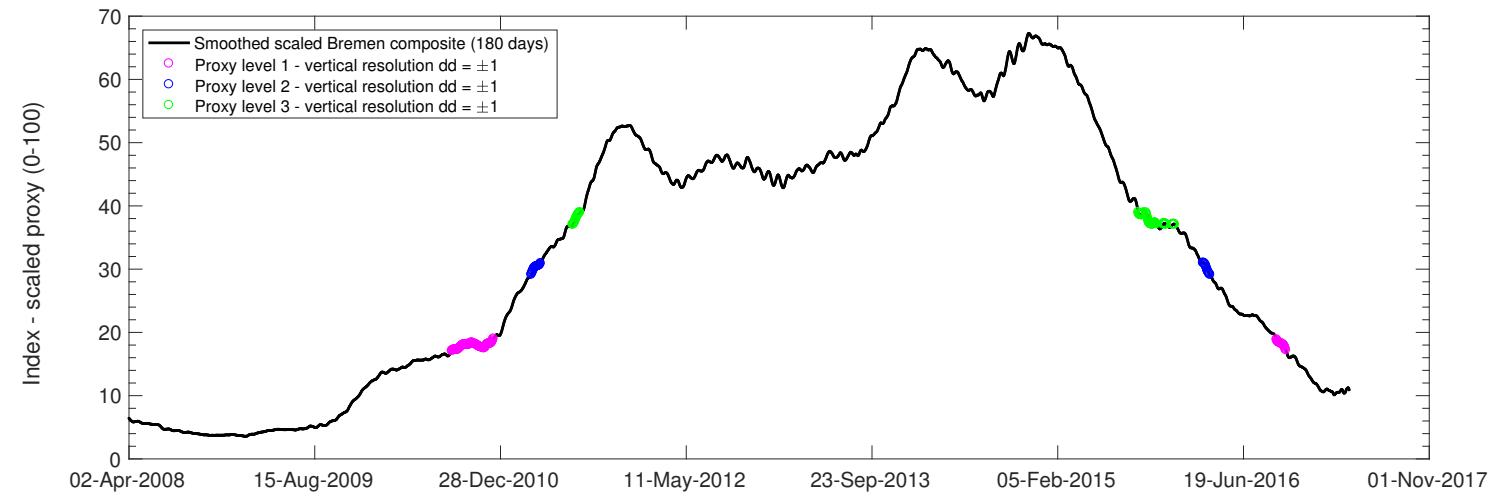

(b)

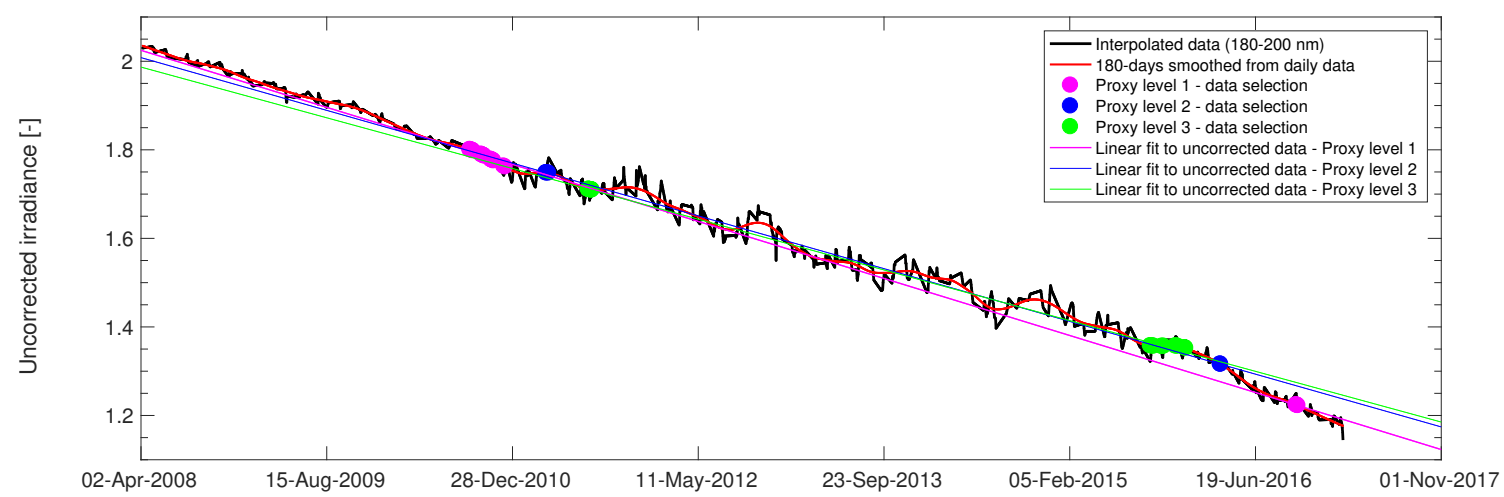

(c)

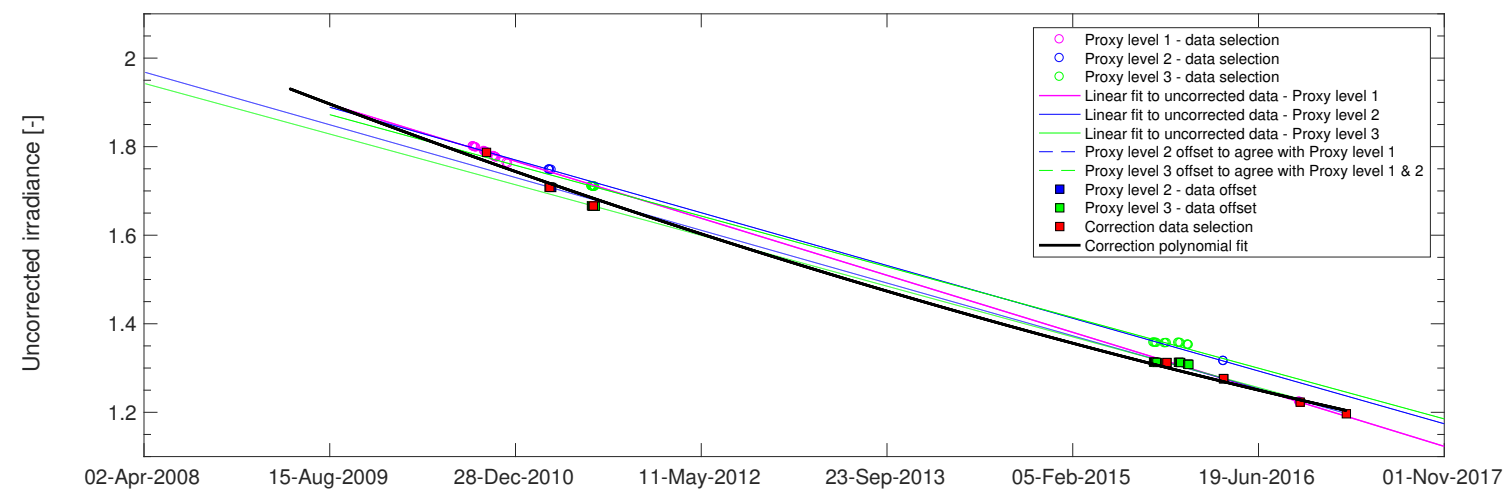

(d)

Fig. 2. SOLAR/SOLSPEC data correction method based on four steps. (a) Step 1: SOLAR/SOLSPEC raw data after preparation. (b) Step 2: Select $n=3$ levels on the steep slopes of the rising and declining phases from smoothed scaled proxy. (c) Step 3: Find times in data corresponding to selected proxy levels. $(d)$ Step 4: Exponential fit to normalized adjusted raw data. 
corresponding to the lowest proxy level and fit a line. This line was taken as the reference trend in the data to be corrected.

The next step was to adjust the data corresponding to the middle proxy value to agree with the reference line on the declining phase of the solar cycle. The simplest method is to determine an additive offset that will force the late mission data to fall on the reference line. After adjusting the data by this offset, we fit a line to the adjusted data (shown as squares in Fig. 2d). The later data corresponding to the highest proxy level (green) were similarly adjusted by an additive offset to lie on the line fit to the adjusted middle proxy level SSI data.

The adjusted data are shown as squares in Fig. 2d. In the final step of our method, we fit a first order exponential to the adjusted data (shown as the black curve in Fig. 2d). This exponential fit (R-square of 0.997) is taken to be the estimated degradation function for the raw SSI data. The statistical uncertainty can be calculated for this fit.

The systematic uncertainty of the method is described in Woods et al. (2018). They used a combination of proxies with an inherent uncertainty of $4 \%$ that was a significant contribution to the final uncertainty of $5 \%$ of the solar cycle. The other primary source of systematic uncertainty is the assumption that the corrected dataset will be linearly correlated with the proxy. Since we only used the Mg II index instead of the super proxy, we employed the Woods et al. (2018) estimate as the upper limit of our uncertainty. The value quoted in Woods et al. (2018) is $5 \%$ of the solar-cycle amplitude, and we adopted this value at each wavelength. For the wavelength band shown in Fig. 3a, the solar-cycle amplitude is about $10 \%$, so the long-term uncertainty of the corrected SOLAR/SOLSPEC data is less than $1 \%$ over solar cycle 24.

Figure $3 \mathrm{~b}$ (left) shows data from Fig. 2a after correction.

\section{Results and discussions}

The modified MuSIL technique was applied to the original SOLAR/SOLSPEC irradiance time series to provide a corrected dataset of relative change in SSI. The approach of the technique is to establish a reference scaled proxy to choose levels on the steep slopes of the rising and declining phases for determining the dates when those same SOLAR/SOLSPEC irradiance levels occur. In the $165-300 \mathrm{~nm}$ wavelength range, the $\mathrm{Mg}$ II index is used since it is the best proxy in this region. Outside of this wavelength range, it would be necessary to use a super proxy that combines sunspot number (photosphere), $\mathrm{Mg}$ II index (chromosphere), $\mathrm{H}_{\text {I }}$ Lyman-alpha (transition region), and F10.7 (corona), as was done in Woods et al. (2018). The selected scaled proxy and the original SOLAR/SOLSPEC irradiance dataset were smoothed over a 180-day boxcar to preserve only the solarcycle variation (remove of the rotational variability and the differences in center-to-limb variation for the solar radiation at different wavelengths). The objective is to enable a more accurate selection of dates that have the same irradiance level. Using the modified MuSIL technique (Sect. 2), we obtained an estimated degradation function for the raw SSI data. The modified MuSIL technique appears to work very well for the far UV and part of the middle UV wavelength bands. At longer than $240 \mathrm{~nm}$, the analysis is more complicated because there is less solar-cycle variability. The uncertainty in the technique becomes a significant fraction of the variation due to the solar cycle. Indeed, mean solar irradiance of recorded SOLAR/SOLSPEC spectra along the mission are distributed in two intensity levels (or, "SOLAR/SOLSPEC families", which depend on operating modes) and a constant ratio exists between them, as shown in
Fig. 1 for the $242-300 \mathrm{~nm}$ wavelength range. These different signal levels in the raw data are due to differences in instrument operation. Observations collected at different parts of the orbit have systematically different temperatures. In this case, SOLAR/SOLSPEC spectra were corrected using a constant ratio between the two kinds of spectra (Fig. 1, see 242-300 nm raw data). This correction allows us to account for the different long-term slopes of measurements. However, it doesn't allow us to account for the different standard deviation of the measured values that after the data preparation continue to show a significantly different spread. The lower irradiance level of the 242-300 nm time series (Fig. 1) is chosen as a reference (arbitrary choice related to the fact that we are only interested in solar variability). In the next subsection, we present the SOLAR/SOLSPEC results on the ultraviolet portion of the SOLAR/SOLSPEC dataset (165-300 nm wavelength range).

\subsection{Results on solar-cycle timescales}

The SOLAR/SOLSPEC SSI time series in four broad wavelength bands spanning 165 through $300 \mathrm{~nm}$ are shown in Fig. 3.

The evolution of each normalized SSI SOLAR/SOLSPEC time series was obtained using the data correction method presented in Sect. 2. These curves correspond to daily interpolated data. Next, we smoothed each SOLAR/SOLSPEC dataset time series with an 81-day running mean to remove higher frequency variability (Fig. 3, left panel). We also compared the SOLAR/SOLSPEC observations with SORCE/SOLSTICE observations, and independent models of SSI variability. The more sophisticated models (semi-empirical) rely on the calculated intensity spectra of magnetic structures on the solar surface and in the solar atmosphere, generated with spectral synthesis codes from semi-empirical solar-model atmospheres. An established example of such models is the Spectral And Total Irradiance REconstruction for the Satellite era (SATIRE-S; Yeo et al. 2017). Another excellent approach is proposed with the Naval Research Laboratory's solar variability model (NRLSSI), which estimates irradiance by using relationships between proxy indicators of solar magnetic variability and solar irradiance observations (Coddington et al. 2019). The SSI variations of the two models are quite close. Between 165 and $300 \mathrm{~nm}$, the NRLSSI model (version 2) shows slightly weaker variations than SATIRE during solar cycle 24, as explained by Coddington et al. (2019). Figure 3 (right panel) shows smoothed SSI time series (81-day running mean) of the SOLAR/SOLSPEC and SORCE/SOLSTICE observations and the SATIRE-S (version date 2019-06-21) and NRLSSI2 (v02 r01, version date 2020-03$02)$ models. These comparisons are made over solar cycle 24 in four broad integrated wavelength bins. For SORCE/SOLSTICE, SATIRE, and NRLSSI, the selected data correspond to observation dates of SOLAR/SOLSPEC measurements to better compare the results.

The right-hand column of Figs. 3a-d show the comparison of our processed SOLAR/SOLSPEC data compared with independent measurements (SORCE/SOLSTICE) and models (SATIRE, NRLSSI). The uncertainty of the SOLAR/SOLSPEC data is shown as the gray shaded area. The SORCE/SOLSTICE, SATIRE, and NRLSSI results fall within the 1-sigma error envelope for wavelengths shorter than $200 \mathrm{~nm}$. In the 200-242 nm band, the SOLAR/SOLSPEC results agree with those of SORCE/SOLSTICE, but the models are nearly $1 \%$ higher (going into the 24/25 minimum) than the two measurements. The solar cycle amplitude in this band is only $3 \%$, so this difference is significant. The $242-300 \mathrm{~nm}$ band for SORCE/SOLSTICE is 

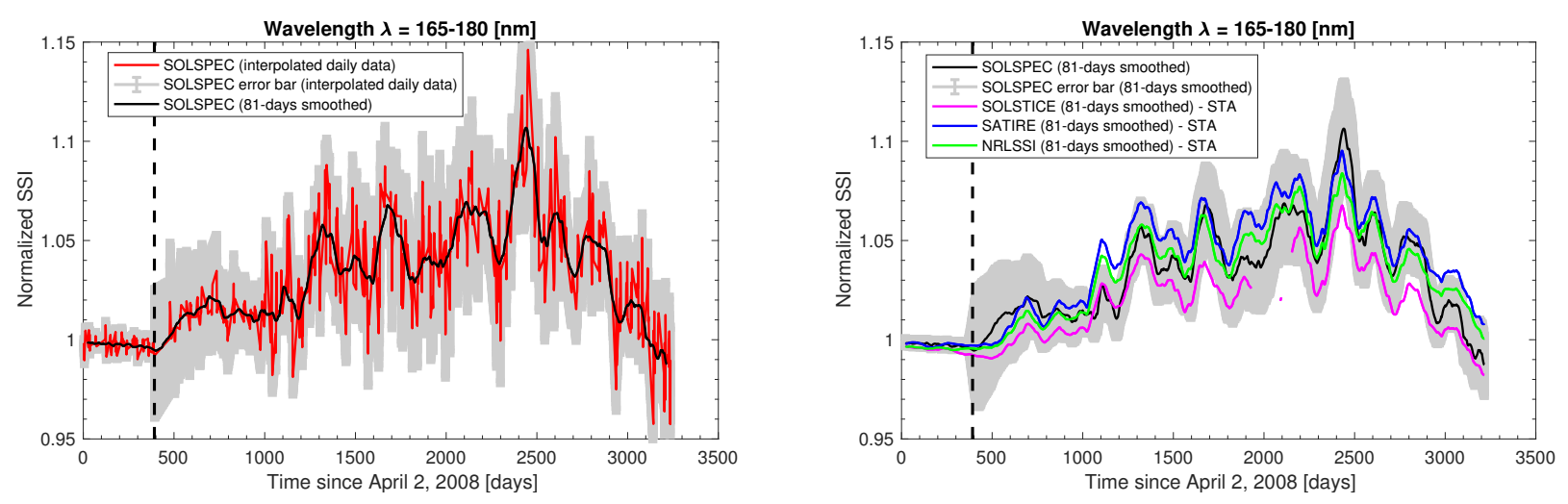

(a)
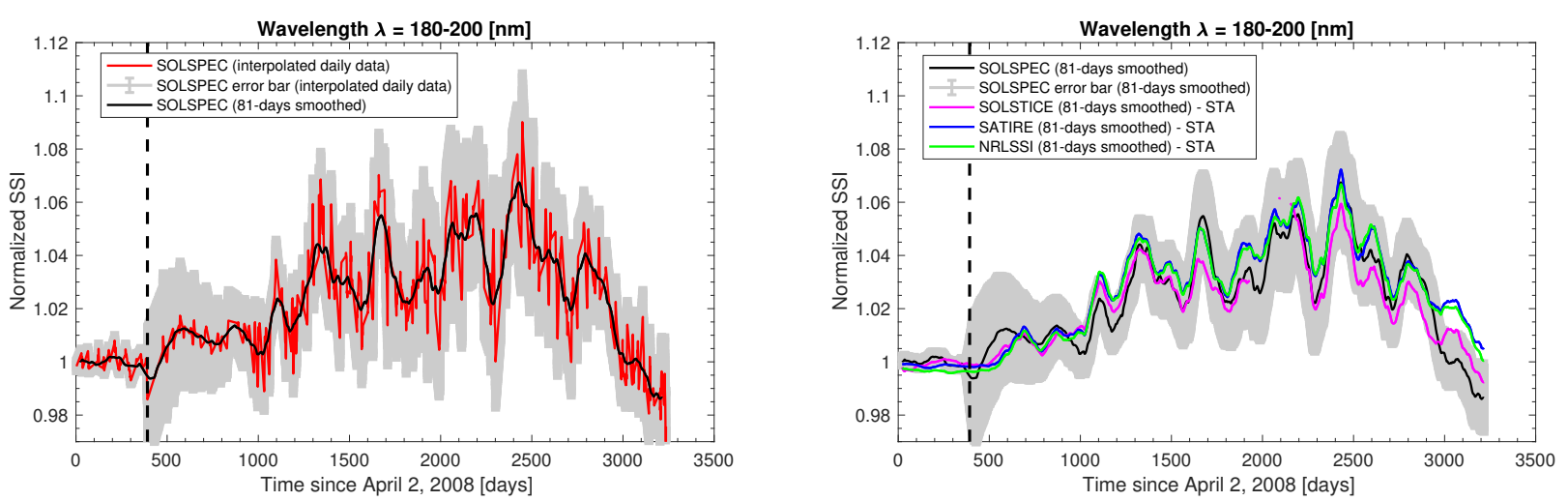

(b)
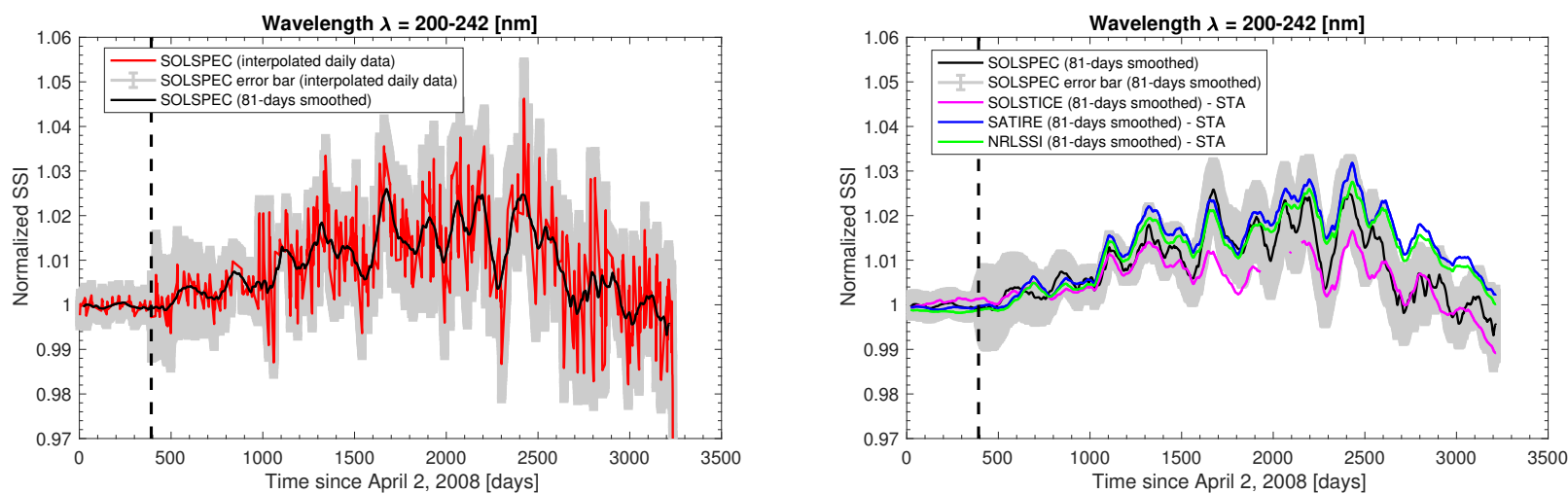

(c)
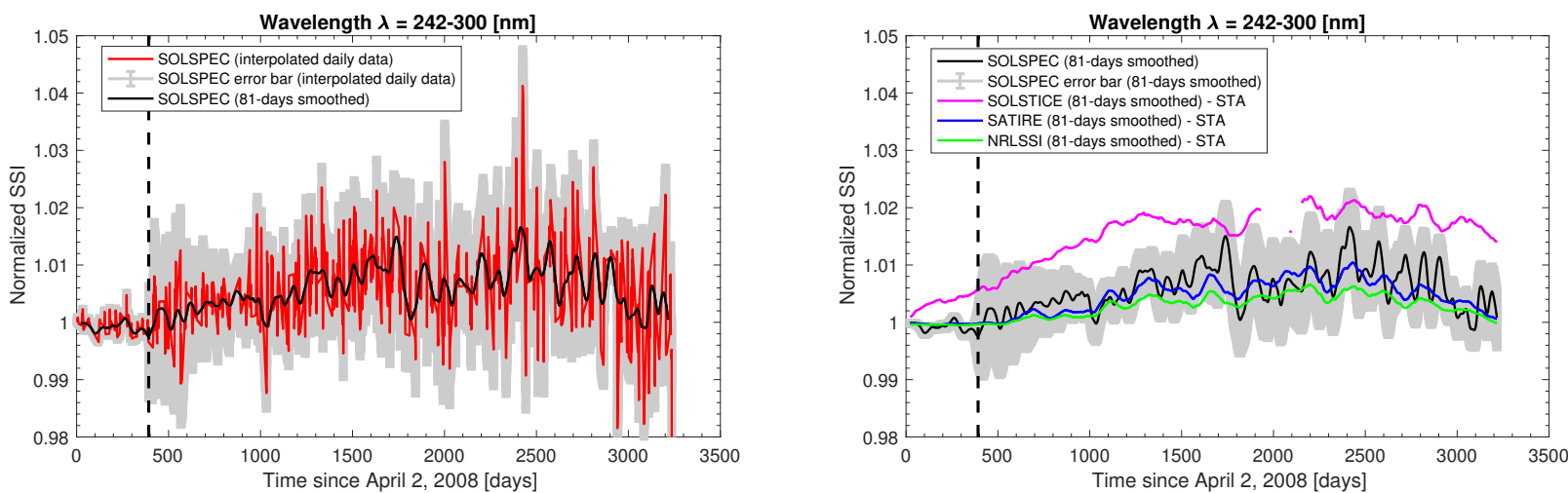

(d)

Fig. 3. SOLAR/SOLSPEC, SORCE/SOLSTICE, SATIRE-S, and NRLSSI 2 normalized solar irradiance in four broad wavelength bands spanning from 165 to $300 \mathrm{~nm}$. SORCE/SOLSTICE, SATIRE-S and NRLSSI 2 SSI data are taken at the SOLAR/SOLSPEC time of acquisition (STA). (a) Irradiance in the $165-180 \mathrm{~nm}$ wavelength range. (b) Irradiance in the 180-200 nm wavelength range. (c) Irradiance in the 200-242 nm wavelength range. $(d)$ Irradiance in the $242-300 \mathrm{~nm}$ wavelength range. 


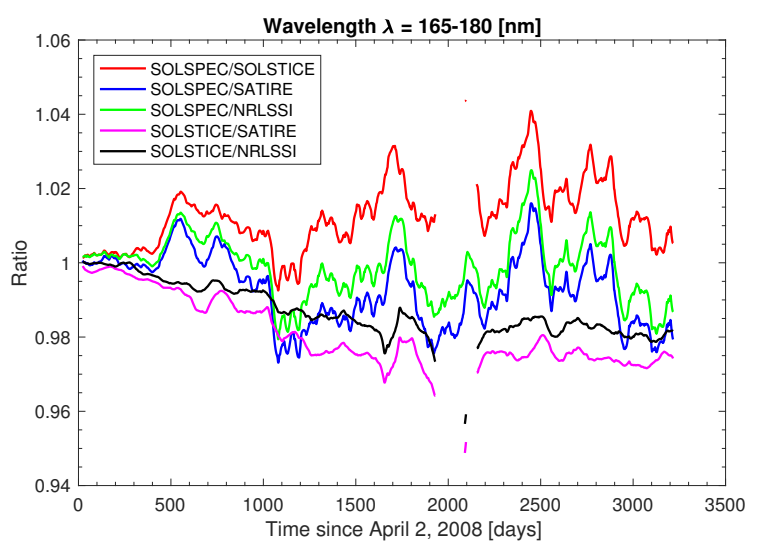

(a)

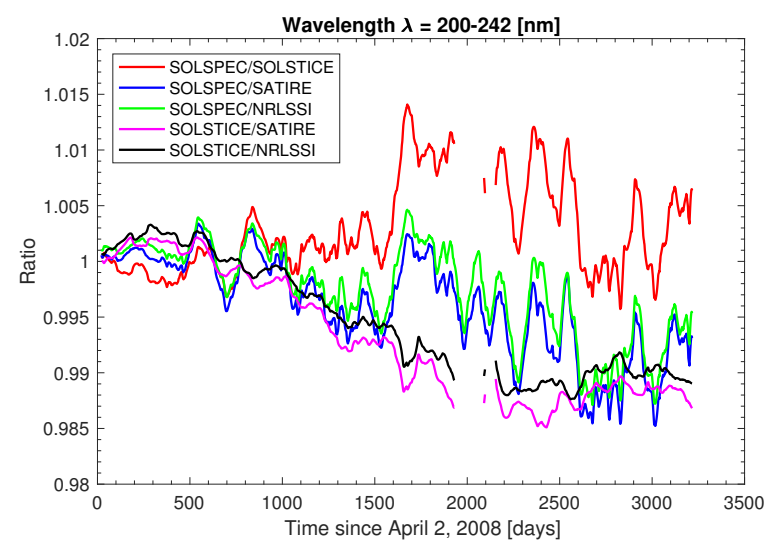

(c)

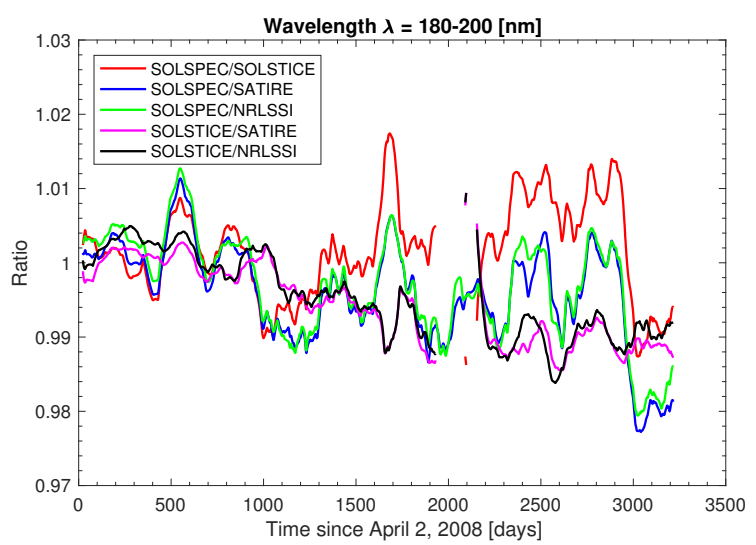

(b)

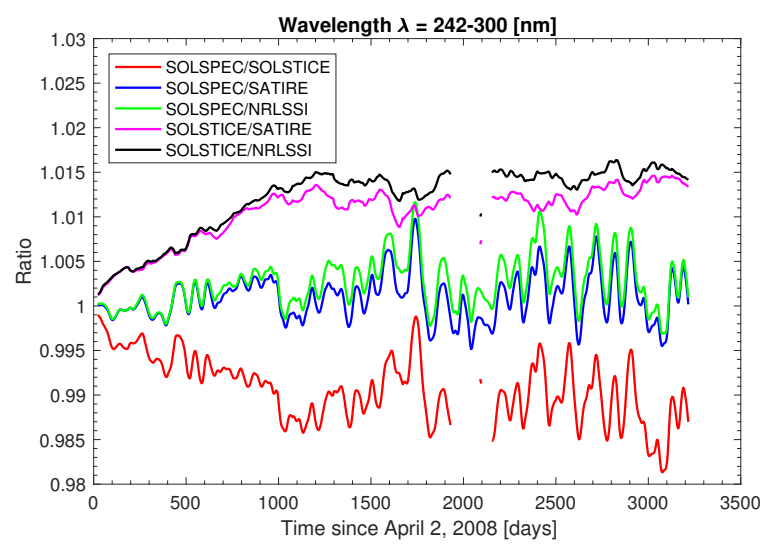

(d)

Fig. 4. SOLSPEC/SOLSTICE, SOLSPEC/SATIRE, SOLSPEC/NRLSSI, SOLSTICE/SATIRE, and SOLSTICE/NRLSSI ratios in four broad wavelength bands spanning 165 through $300 \mathrm{~nm}$. All datasets have been smoothed with an 81-day running mean. (a) $165-180 \mathrm{~nm}$. (b) $180-200 \mathrm{~nm}$. (c) 200-242 nm. (d) 242-300 nm.

dominated by the $290-300 \mathrm{~nm}$ irradiance that has a poor degradation correction (Harder et al. 2009). In that wavelength band (Fig. 3d), the SOLAR/SOLSPEC data agree with the models to within the 1-sigma error envelope.

For comparing the various sets of data (observations and solar models), SOLSPEC/SOLSTICE, SOLSPEC/SATIRE, SOLSPEC/NRLSSI, SOLSTICE/SATIRE, and SOLSTICE/ NRLSSI ratios are plotted in Fig. 4. Between 165 and $180 \mathrm{~nm}$, the differences can go up to $4 \%$ at a given time. Differences between SOLAR/SOLSPEC and the solar models are of around $\pm 2 \%$. Between 180 and $200 \mathrm{~nm}$, the differences of the various sets of data are close to $2 \%$. Between 200 and $242 \mathrm{~nm}$, the differences of the datasets are as close to $1 \%$ as between 242 and $300 \mathrm{~nm}$. The SOLAR/SOLSPEC dataset is sometimes in excellent agreement with the models, and other times with the observations. Observations with space-based instruments provide the unique absolute values of the reference solar spectrum at a given time. Models can be effective in monitoring variability. The main point is to compare the trend of anomalies and the difference between models and observation. In general, SOLAR/SOLSPEC agrees in magnitude with SORCE/SOLSTICE and models during solar cycle 24 and within the SOLAR/SOLSPEC uncertainties.

Figure 5 shows the solar-cycle variability after the analysis corrections were applied for the SOLAR/SOLSPEC SSI dataset. At $165 \mathrm{~nm}$, the SOLAR/SOLSPEC SSI variability during a solar cycle is close to $16 \%$. At $300 \mathrm{~nm}$, the SOLAR/SOLSPEC SSI variability during a solar cycle is close to $1 \%$. For wavelengths greater than $300 \mathrm{~nm}$, the SOLAR/SOLSPEC precise determination of the solar variability remains complex. The primary purpose of solar irradiance models is to provide estimated SSI variability using the solar proxies and based on solar observations obtained at the top of the atmosphere with space-based instruments. There are also several irradiance models that make use of ground-based observations, specifically the ones presented by Fontenla et al. (1999), Ermolli et al. (2003, 2011), Criscuoli et al. (2018), Fontenla \& Landi (2018), and Chatzistergos et al. (2020).

\subsection{Solar spectral irradiance on solar rotational timescales and high frequency}

The UV irradiance of the SOLAR/SOLSPEC instrument compares well with rotational variability in the observations from SORCE/SOLSTICE and models (SATIRE and NRLSSI). The SOLAR/SOLSPEC captures SSI on solar rotational timescales. Figures 6 and 7 present an investigation of the main features of the estimated UV SSI at two spectral ranges.

The Lomb-Scargle periodogram approach is used to detect periodic signals in unevenly-spaced observations of the SOLAR/ SOLSPEC instrument. Figure 6 shows the SOLAR/SOLSPEC Lomb-Scargle normalized periodograms for the $180-200 \mathrm{~nm}$ wavelength range (as an example) and for the full period of observation of SOLAR/SOLSPEC from 2008 to 2017 


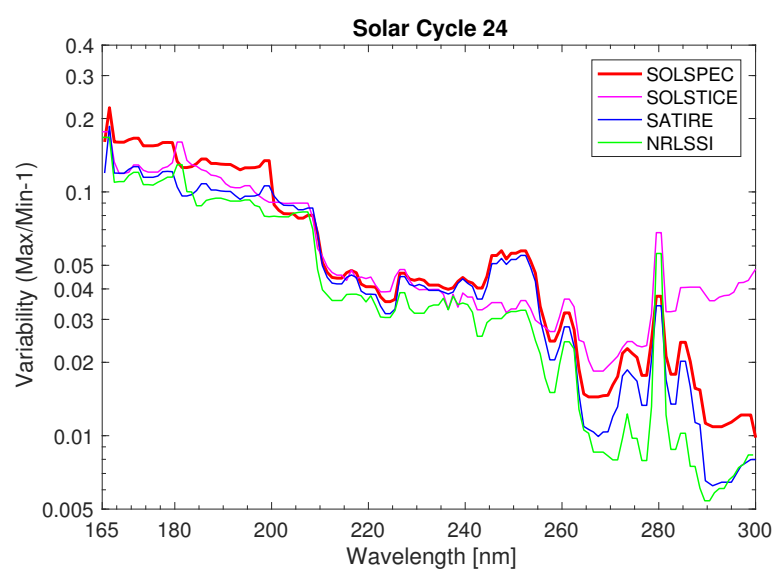

Fig. 5. Solar cycle 24 variability for observations (SOLAR/SOLSPEC and SORCE/SOLSTICE) and models (SATIRE and NRLSSI). The time range of solar cycle 24 is the time from the minimum of cycle $23 / 24$ to the maximum of solar cycle 24 (late 2008 to early 2015).

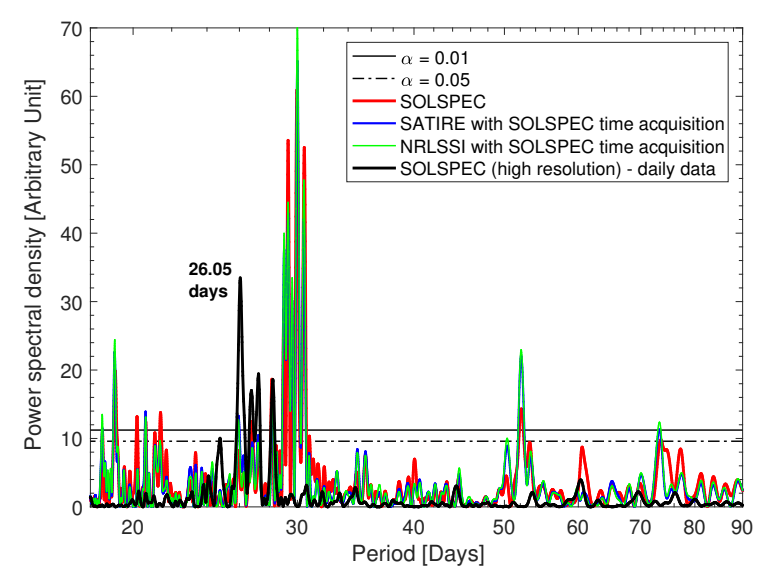

Fig. 6. SOLAR/SOLSPEC (red), SATIRE (blue), and NRLSSI (green) Lomb-Scargle normalized periodograms $(180-200 \mathrm{~nm}$ wavelength range) from the same unevenly-spaced observations. The black curve represents SOLAR/SOLSPEC data (high resolution) oversampled at the SATIRE temporal resolution (daily data). Statistical significances $(\alpha)$ are given for $95 \%$ and $99 \%$ confidence intervals.

without failure. The statistical significance $(\alpha)$ is also provided for each value of the Lomb-Scargle normalized periodogram. The comparison between SOLAR/SOLSPEC and SATIRE (with the same unevenly spaced observations of SOLAR/SOLSPEC) is consistent. The comparison between SOLAR/SOLSPEC and NRLSSI is also consistent. For unevenly spaced SOLAR/SOLSPEC observations such as for SATIRE and NRLSSI (with a SOLAR/SOLSPEC time acquisition), the significant synodic period is around 30 days for the $180-200 \mathrm{~nm}$ wavelength range during solar cycle 24 . This result shows that the solar periods obtained are slightly biased and that daily data sampling is necessary. No comparison was made with SORCE/SOLSTICE, because there is a gap in the SOLSTICE data from July 16, 2013 to February 24, 2014 due to a battery anomaly of the SORCE spacecraft. During this period, SOLAR/SOLSPEC was the only space-based instrument to capture SSI at the top of the atmosphere.

In order to obtain daily data, we multiplied the SSI of the solar model data (SATIRE) by the ratio of the smoothed SSI time series of the SOLAR/SOLSPEC (81-day running mean) to the smoothed SSI time series of the solar model (81-day

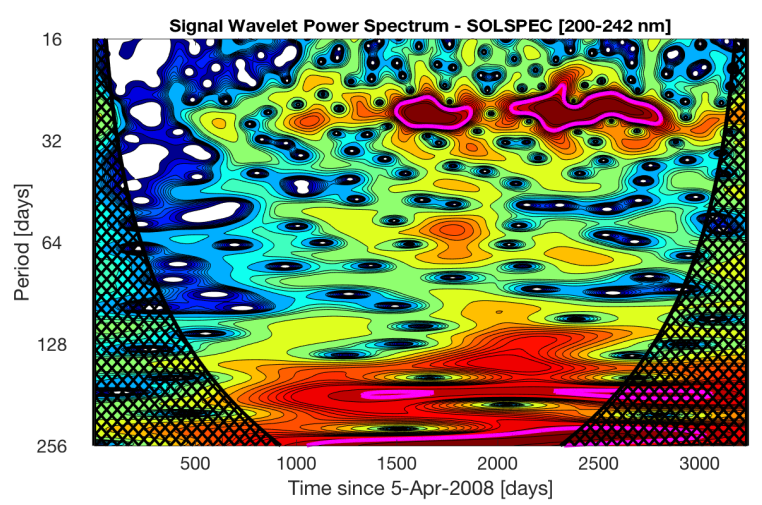

Fig. 7. Signal wavelet power spectrum from Morlet wavelet analysis of SOLAR/SOLSPEC time series (200-242 nm). The plot is jet-colorcoded, where bright red indicates high wavelet power spectrum values. The regions with greater than $95 \%$ confidence are shown with thin pink contours. Anything below the cone of influence is dubious.

running mean). The SOLAR/SOLSPEC corrected time series (Fig. 6, black curve) highlights the significant and realistic synodic period that is close to 26 days for the $180-200 \mathrm{~nm}$ wavelength range during solar cycle 24 . For the other broad wavelength band, the detected synodic period is the same.

For multiple reasons, it is necessary to have SSI time series (observations and models) with high temporal resolution (daily data at least). At this cadence, a time-frequency analysis of solar spectral irradiance is possible. Morlet wavelet spectra clearly and comprehensively characterize the spectral composition of SSI data as well as its temporal changes displaying both non-stationary behavior and recurrent periodicities. Figure 7 shows the time-frequency analysis of the SOLAR/SOLSPEC SSI (200-242 nm) time-series data. With this type of analysis, we can extend our research and carry out cross-wavelet analysis of the relationship between SOLAR/SOLSPEC SSI and a given parameter (sunspot number, Mg II index, H I Lyman-alpha, F10.7, but also stratospheric ozone). A new solar spectral irradiance database (SOLAR-v) is now available to undertake, among others, this kind of studies during solar cycle 24 . One of our objectives is to improve our quantitative understanding of the effects of solar irradiance variability on the atmosphere and climate such as for example the sensitivity of the tropical stratospheric ozone response (Thiéblemont et al. 2017) to the solar rotation during solar cycle 24 in observations and chemistryclimate model simulations. Figure 8 shows SSI time series (absolute scale) in four narrow wavelength bands.

\section{Conclusions}

In this work, we developed a new method based on the MuSIL technique to correct SOLAR/SOLSPEC data. We established that there is consistency between the SOLAR/SOLSPEC SSI and the observational record (SORCE/SOLSTICE), and also with the solar models (SATIRE and NRLSSI). The uncertainty in this comparison is tied to the inherent uncertainty of the MuSIL technique, which is estimated to be $5 \%$ of the solar-cycle amplitude (Woods et al. 2018). This uncertainty comes from several sources in Woods et al. (2018), including the standard deviation between the elements of their "super proxy." Our study uses only the Mg II index, which is the most appropriate proxy for the ultraviolet (Dudok de Wit et al. 2009). Woods et al. (2018) attributed an uncertainty of $4 \%$ to variations among the super proxy components, so ours only includes that of the Mg II index, 

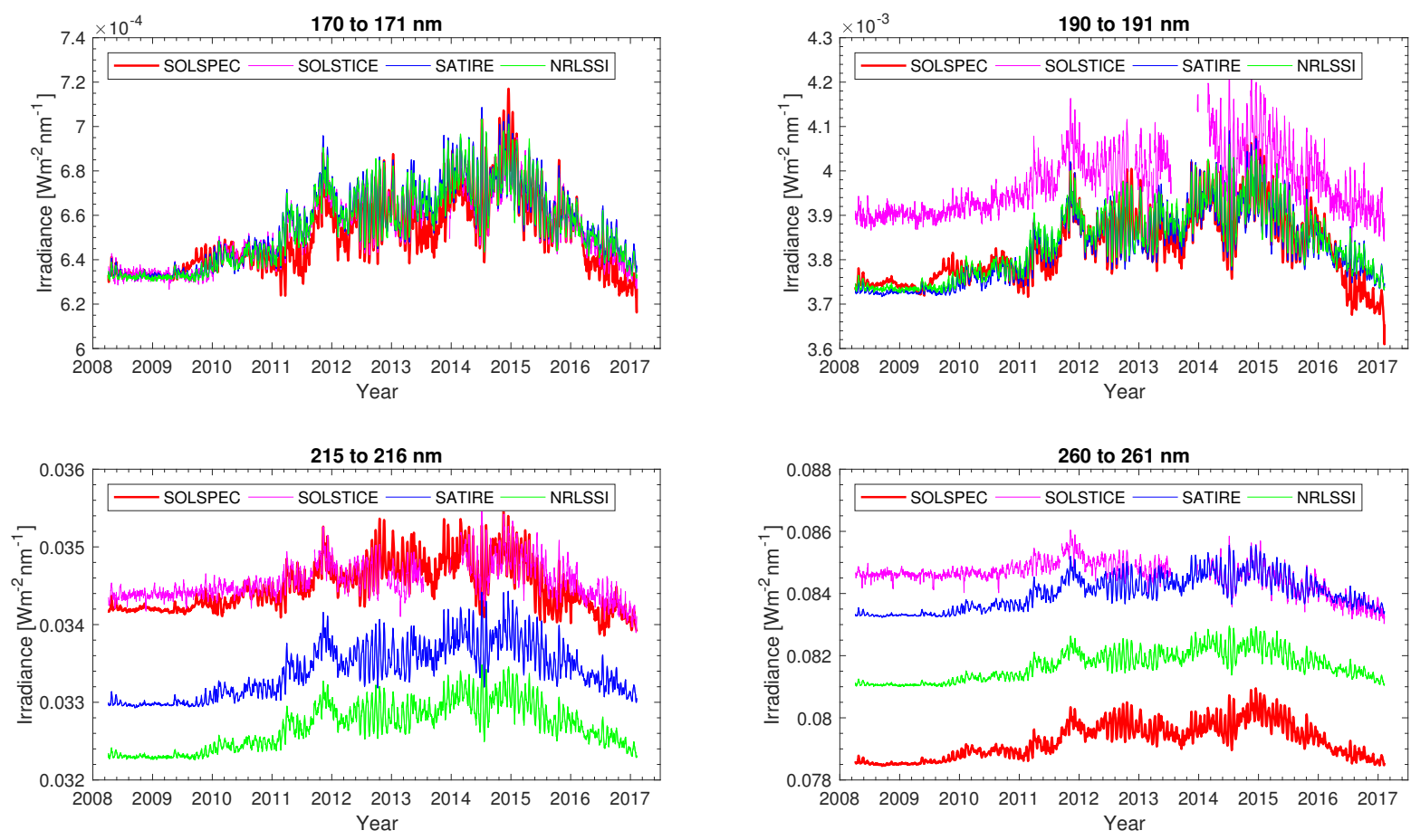

Fig. 8. SOLAR/SOLSPEC, SORCE/SOLSTICE, SATIRE, and NRLSSI solar irradiance in four narrow wavelength bands.

which is estimated to have an uncertainty of less than $1 \%$ of the solar-cycle amplitude (Snow et al. 2019). Therefore, we cite the $5 \%$ uncertainty as an upper limit to our estimated one.

Space-based spectrometers are fundamental for providing SSI absolute scales and for measuring variability for wavelengths less than $300 \mathrm{~nm}$ (resolution less than $1 \mathrm{~nm}$ ), where continuity of measurements is crucial over multiple solar cycles. The new SOLAR/SOLSPEC database and future space-based UV observations are necessary for chemistry-climate models.

To conclude, the SOLAR-v data record provides a new important scientific database of solar irradiance that is of sufficient length, consistency, and continuity for use in studies of climate variability and climate change on multiple time scales. The SOLAR-v data record (Level 3, V1.0) is available at the Centre de Données astronomiques de Strasbourg.

Acknowledgements. This work was supported by Centre National de la Recherche Scientifique (CNRS, France), the Royal Belgian Institute for Space Aeronomy (BIRA-IASB, Belgium), the European Space Agency (ESA, Europe), and the Centre National d'Études Spatiales (CNES, France). MS was supported NASA contract NAS5-97045 (SORCE) at the University of Colorado (USA) This work is based on observations with SOLAR/SOLSPEC embarked onboard the international space station. We are grateful to the instrument teams for providing their public data products for this study. The SOLAR team gratefully acknowledges Astrid Orr (ESA), Denis Jouglet (CNES), Charlotte Revel (CNES) and the SOLAR team for their support in the implementation of the new SOLAR $\mathrm{v}$ data record. The authors would like to thank the anonymous reviewer for useful suggestions throughout this study and for the thoughtful comments. This work is useful for the preparation of the UVSQ-SAT (Meftah et al. 2019) and MicroCarb missions.

\section{References}

BenMoussa, A., Gissot, S., Schühle, U., et al. 2013, Sol. Phys., 288, 389 Blunden, J., \& Arndt, D. S. 2014, Bull. Am. Meteorol. Soc., 95, S1 Bolsée, D., Pereira, N., Gillotay, D., et al. 2017, A\&A, 600, A21

Chatzistergos, T., Ermolli, I., Giorgi, F., Krivova, N. A., \& Puiu, C. C. 2020, J. Space Weather Space Clim., 10, 45

Coddington, O., Lean, J., Pilewskie, P., et al. 2019, Earth Space Sci., 6, 2525

Criscuoli, S., Penza, V., Lovric, M., \& Berrilli, F. 2018, ApJ, 865, 22
Dudok de Wit, T., Kretzschmar, M., Lilensten, J., \& Woods, T. 2009, Geophys. Res. Lett., 36, L10107

Ermolli, I., Caccin, B., Centrone, M., \& Penza, V. 2003, Mem. Soc. Astron. It., 74, 603

Ermolli, I., Criscuoli, S., \& Giorgi, F. 2011, Contrib. Astron. Obs. Skaln. Pleso, 41,73

Ermolli, I., Matthes, K., Dudok de Wit, T., et al. 2013, Atm. Chem. Phys., 13, 3945

Floyd, L., Tobiska, W. K., \& Cebula, R. P. 2002, Adv. Space Res., 29, 1427

Fontenla, J. M., \& Landi, E. 2018, ApJ, 861, 120

Fontenla, J., White, O. R., Fox, P. A., Avrett, E. H., \& Kurucz, R. L. 1999, ApJ, 518,480

Gray, L. J., Beer, J., Geller, M., et al. 2010, Rev. Geophys., 48, RG4001

Haigh, J. D. 1994, Nature, 370, 544

Harder, J., Lawrence, G., Fontenla, J., Rottman, G., \& Woods, T. 2005, Sol. Phys., 230, 141

Harder, J. W., Fontenla, J. M., Pilewskie, P., Richard, E. C., \& Woods, T. N. 2009, Geophys. Res. Lett., 36, 7801

Heath, D. F., \& Schlesinger, B. M. 1986, J. Geophys. Res., 91, 8672

Kren, A. C., Pilewskie, P., \& Coddington, O. 2017, J. Space Weather Space Clim., 7, A10

Marchenko, S. V., DeLand, M. T., \& Lean, J. L. 2016, J. Space Weather Space Clim., 6, A40

McClintock, W. E., Rottman, G. J., \& Woods, T. N. 2005, Sol. Phys, 230, 225

Meftah, M., Bolsée, D., Damé, L., et al. 2016, Sol. Phys., 291, 3527

Meftah, M., Dominique, M., BenMoussa, A., et al. 2017a, in SPIE Conf. Ser. Proc. SPIE, 10196, 1019606

Meftah, M., Damé, L., Bolsée, D., et al. 2017b, Sol. Phys., 292, 101

Meftah, M., Damé, L., Bolsée, D., et al. 2018, A\&A, 611, A1

Meftah, M., Damé, L., Keckhut, P., et al. 2019, Remote Sens., 12, 92

Meftah, M., Damé, L., Bolsée, D., et al. 2020, Sol. Phys., 295, 14

Rottman, G. 2005, Sol. Phys., 230, 7

Shindell, D. T., Faluvegi, G., \& Schmidt, G. A. 2020, J. Geophys. Res.: Atm., 125, e 31640

Snow, M., Weber, M., Machol, J., Viereck, R., \& Richard, E. 2014, J. Space Weather Space Clim., 4, A04

Snow, M., Machol, J., Viereck, R., et al. 2019, Earth Space Sci., 6, 2106

Solanki, S. K., Krivova, N. A., \& Haigh, J. D. 2013, ARA\&A, 51, 311

Thiéblemont, R., Marchand, M., Bekki, S., et al. 2017, Atm. Chem. Phys., 17, 9897

Thuillier, G., Foujols, T., Bolsée, D., et al. 2009, Sol. Phys., 257, 185

Woods, T. N., Eparvier, F. G., Harder, J., \& Snow, M. 2018, Sol. Phys., 293, 76

Yeo, K. L., Krivova, N. A., \& Solanki, S. K. 2017, J. Geophys. Res.: Space Phys., 122, 3888 